\title{
Different Symmetry Realizations in Relativistic Coupled Bose Systems at Finite Temperature and Densities
}

\author{
R. L. S. Farias, R. O. Ramos, and R. Vartuli \\ Departamento de Física Teórica, Universidade do Estado do Rio de Janeiro, 20550-013 Rio de Janeiro, RJ, Brazil
}

(Received on 17 April, 2008)

\begin{abstract}
We revisited the calculation of the effective potential for self-interacting scalar field with $U(1)$ charge at one loop approximation. We show that high charge densities can induce important changes in the phase structure of the theory. A class of very interesting phenomena appear when we introduce finite density effects, e.g. symmetry nonrestoration, inverse symmetry breaking and anticipation of the high temperature symmetry restoration. The extension of these calculations in the context of multi-scalar field theory is outlined, with the objectives of studying the effects of a finite charge on the symmetry breaking phase transition, and to learn how these effects change the number of phases allowed by the system symmetries.
\end{abstract}

Keywords: Relativistic heavy-ion collisions; Inverse symmetry breaking and symmetry nonrestoration

\section{INTRODUCTION}

Much work has been done exploring how field theories behave at finite temperature and densities [1-4]. The study of symmetry breaking (SB) and symmetry restoration (SR) mechanisms have proved to be extremely useful in the analysis of phenomena related to phase transitions in almost all branches of physics [5]. For a large number of physical systems we have a good idea, both qualitatively as quantitatively of how symmetries change as the temperature is changed. Usually, we expect that the larger is the temperature the larger is the symmetry manifested by the system and vice-versa. This behavior is expected in high energy systems like particle physics models (e.g. in the electroweak phase transition) and in lower energy systems like ones found in condensed matter (e.g. Bose-Einstein condensation in atomic gases).

An almost general rule that arises from studies of how the symmetry changes with temperature is that a symmetry which is broken at zero temperature should get restored as the temperature increases. Examples range from the traditional ferromagnet to the more up to date chiral symmetry breaking/restoration in Quantum chromodynamics (QCD), with the transition pattern being the simplest one of going from the broken phase to the symmetric one as temperature goes from below to above some critical value and vice-versa. One very interesting counter example was shown by Weinberg in the context of multiscalar field theories at finite temperatures [6]. He has shown the possibility of appearing two very interesting phenomena: one that occurs when a symmetry that is not broken at low temperatures, can get broken at high temperatures, a phenomenon called inverse symmetry breaking (ISB), and the possibility of another case, that can happen when a symmetry that is broken at lower temperatures, may never get restored at all as we go to higher temperatures, a phenomenon called symmetry nonrestoration (SNR).

These phenomena of ISB and SNR can be found in the context of high energy physics due to possibility of their implementation in realistic particle physics models, like in the context of high temperature phase transitions in the early Universe $[7,8]$ and in applications covering problems which involve $\mathrm{CP}$ violation and baryogenesis, topological defect formation, inflation, etc [9]. Beside these interesting applica- tions, there are real physical systems which do exhibit phenomena similar to ISB/SNR, like liquid crystals, spin glass materials and many other systems and materials [10]. One of us have recently analyzed how ISB/SNR manifest themselves in nonrelativistic theories which may be used in condensed matter physics $[8,11]$ and possible applications of ISB/SNR phenomena to a coupled two-species dilute Bose gas system $[12,13]$.

In this paper we are looking for the phenomena of symmetry nonrestoration and inverse symmetry breaking in the context of multi-scalar field theories considering the effects of both finite temperature and density. In other words we are interested in the different symmetries realizations and the effects of a finite charge on a multi-scalar field theory. After reviewing the results at finite temperature for a coupled twoscalar field model, we give the results obtained for a scalar self-interacting theory with a fixed $U(1)$ charge [14] at finite temperature. The extension of these results and calculations for a multi-scalar field theory at both finite temperature and densities are then explained. Our aim here is try to understand how a nonzero charge affects the phase structure of a multiscalar field theory, which should be of relevance in processes that may occur in heavy-ion collisions and in the early universe.

\section{MULTI-SCALAR FIELD THEORY: LOOKING FOR ISB AND SNR}

Let us initially briefly review the symmetry broken (SB) / symmetry restoration (SR) for a self-interacting real scalar field theory at finite temperature. Then, we contrast these results with those that may originate as a consequence of having more than one scalar field with cross-couplings showing how phenomena ISB or SNR can emerge.

\subsection{Symmetry breaking/restoration in $O(N)$ scalar models}

We start with the case of a relativistic case self-interacting scalar field with a $O(N)$ symmetry. The Lagrangian density is given by 


$$
\mathcal{L}(\phi)=\frac{1}{2}(\partial \phi)^{2}-V(\phi),
$$

where $\phi$ is a scalar field with $N$ components and the potential $V(\phi)$ is given by

$$
V(\phi)=\frac{1}{2} m^{2} \phi^{2}+\frac{\lambda_{\phi}}{4 !} \phi^{4}
$$

This potential is bounded if $\lambda>0$ and depending on the signal of the mass term we have the system either in the unbroken phase, $\left(m^{2}>0\right)$, or broken phase, $\left(m^{2}<0\right)$. By considering initially the broken phase at $T=0$, we have for instance that

$$
V(\phi, T=0)=-\frac{1}{2} m^{2} \phi^{2}+\frac{\lambda_{\phi}}{4 !} \phi^{4}
$$

for which there is a nonvanishing vacuum expectation value. At finite temperature the potential acquires thermal corrections and, for example at high temperatures $[2,6]$, it (the effective potential) acquires the following form

$$
V(\phi, T \neq 0) \simeq \frac{1}{2}\left[-m^{2}+\lambda_{\phi} \frac{(N+2) T^{2}}{72}\right] \phi^{2}+\frac{\lambda_{\phi}}{4 !} \phi^{4} .
$$

From the above expression we obtain that there is a critical temperature $T_{c}$ above which the vacuum expectation value for the effective potential vanishes and the symmetry is restored, with $T_{c}$ given by

$$
T_{c}=\sqrt{\frac{72 m^{2}}{(N+2) \lambda}}
$$

It is very interesting to mention that, in the perturbative regime, the dominant contribution giving $T_{c}$ comes from a one-loop correction, and it is not expected to change too much due to higher order contributions.

\section{2. $O(N) \times O(N)$ relativistic models}

Having shown the simple one field case in the previous subsection, let us see now how it is possible to appear different symmetry breaking/restoring patterns, like ISB and/or SNR. These phenomena were first shown possible in finite temperature quantum field theory by Weinberg in 1974 [6]. Weinberg proposed the following multi-scalar field model (in either the unbroken or broken phase initially for both the $\phi$ and $\psi$ directions, depending on the sign in front of the mass terms),

$$
\begin{aligned}
\mathcal{L}(\phi, \psi) & =\frac{1}{2}\left(\partial_{\mu} \phi\right)^{2}-\frac{m_{\phi}^{2}}{2} \phi^{2}-\frac{\lambda_{\phi}}{4 !}\left(\phi^{2}\right)^{2}+\frac{1}{2}\left(\partial_{\mu} \psi\right)^{2} \\
& -\frac{m_{\psi}^{2}}{2} \psi^{2}-\frac{\lambda_{\psi}}{4 !}\left(\psi^{2}\right)^{2}-\frac{\lambda}{4} \phi^{2} \psi^{2}
\end{aligned}
$$

The potential that appear in this Lagrangian density is bounded from below for $\lambda_{\phi}>0, \lambda_{\psi}>0$ and $\lambda_{\phi} \lambda_{\psi}>9 \lambda^{2}$. One important aspect here is that we can change the signal of the coupling constant $\lambda$ and the potential still remain bounded.

The thermal masses $M_{\phi}^{2}(T)$ and $M_{\psi}^{2}(T)$ obtained from Eq. (6) can be readily obtained [7],

$$
\begin{gathered}
M_{\phi}^{2}(T)=m_{\phi}^{2}+\Sigma_{\phi}^{T}, \\
M_{\psi}^{2}(T)=m_{\psi}^{2}+\Sigma_{\psi}^{T},
\end{gathered}
$$

where $\Sigma_{\phi}^{T}$ and $\Sigma_{\psi}^{T}$ are the self-energies related to the fields $\phi$ and $\psi$, respectively. We then obtain the thermal masses $M_{\phi}^{2}(T)$ and $M_{\psi}^{2}(T)$ as given, in the high temperature approximation, by

$$
\begin{aligned}
& M_{\phi}^{2}=m_{\phi}^{2}+\frac{T^{2}}{24}\left(\lambda_{\phi} \frac{N+2}{3}+\lambda N\right), \\
& M_{\psi}^{2}=m_{\psi}^{2}+\frac{T^{2}}{24}\left(\lambda_{\psi} \frac{N+2}{3}+\lambda N\right) .
\end{aligned}
$$

For $N=2$ for example and using (9) and (10), we obtain the critical temperature $(i=\phi, \psi)$

$$
T_{c, i}=\left[-12 m_{i}^{2}\left(\lambda_{i} \frac{2}{3}+\lambda\right)^{-1}\right]^{1 / 2} .
$$

Assuming $\lambda<0$, or in other words, $|\lambda|>2 \lambda_{\phi} / 3$, remembering that the boundness condition assures that $|\lambda|<2 \lambda_{\psi} / 3$. We note that by taking $m_{i}^{2}<0$ and using the equations (10) and (11), in the $\psi$ sector we have SR, but in the $\phi$ sector we have SNR. In the opposite case, where $m_{i}^{2}>0$, in the $\psi$ sector the system remains in the unbroken phase, while in the $\phi$ sector we have ISB.

As this simple example illustrates, we note that temperature effects in multiscalar field models can change the symmetry aspects in unexpected ways, e.g., in the $O(N) \times O(N)$ example, it shows the possibilities of phenomena like inverse symmetry breaking (ISB) and symmetry nonrestoration (SNR). One very interesting question that appears is: Can we trust perturbative methods at high temperatures? Though we know that high temperature field theories require nonperturbative treatments for consistence [7], it has been shown extensively in the literature that these phenomena also appear in nonperturbative approaches, thus they are not artifacts of perturbation theory. One discussion in a fully nonperturbative context of the phenomena of SNR and ISB was done in Ref. [15] (see also references therein for other nonperturbative approaches).

We can say that for a relativistic $O(N) \times O(N)$ theory, ISB/SNR phenomena is possible. Many interesting application of these phenomena have been proposed in the literature, like in cosmology, in the context of formation of monopoles/domain walls and also in condensed matter physics, Ref. [10], where several applications were discussed. 


\section{PHASE STRUCTURE AND THE EFFECTIVE POTENTIAL AT FIXED CHARGE}

Having briefly discussed the effects of temperature in symmetry restoring/breaking of symmetries, let us now see how density effects can also affect the symmetry properties of a theory. Let us briefly see the derivation of the effective potential for a self-interacting scalar field theory at finite temperature and density (with a fixed $U(1)$ charge). For simplicity we restrict the calculation at the one-loop order $[1,2,14,16]$. We start with the grand partition function,

$$
Z\left(\beta, \Omega, J_{i}, \mu\right) \equiv \operatorname{Tr}\left\{\exp \left[-\beta\left(\mathcal{H}^{\prime}-\mu Q\right)\right]\right\},
$$

where $\beta$ is the inverse of the temperature, $\Omega$ is the volume of the system, $J_{i}$ are the external sources, $\mu$ is the chemical potential, $\mathcal{H}$ is the Hamiltonian density and $\mathcal{H}^{\prime}$ is given by

$$
\mathcal{H}^{\prime}=\mathcal{H}-\frac{J_{i}}{\beta \Omega} \int d^{3} x \phi_{i}(x)
$$

Using standard manipulations [14] like Legendre transformations we obtain the one-loop effective potential,

$$
V_{\mathrm{eff}}=-\frac{1}{\beta \Omega}\left(\ln (Z)+J_{i} \phi_{i}\right)+\frac{\mu \bar{Q}}{\Omega},
$$

where $\bar{Q}$ is a charge constraint

$$
\bar{Q} \equiv\langle Q\rangle=\frac{1}{\beta} \frac{\partial}{\partial \mu} \ln Z .
$$

Rewriting the trace of Eq.(12) as a functional integral (the index $i$ runs from 1 and 2) we get

$$
\begin{aligned}
Z\left[J_{i}\right] & =\int \mathcal{D} \phi_{1} \mathcal{D} \phi_{2} \mathcal{D} \pi_{1} \mathcal{D} \pi_{2} \exp \left\{\int _ { 0 } ^ { \beta } d \tau \int d ^ { 3 } x \left[i\left(\pi_{i} \dot{\phi}_{i}\right)\right.\right. \\
& -(\mathcal{H}-\mu Q)]\}
\end{aligned}
$$

The functional integral above is over two real fields $\left(\phi_{1}, \phi_{2}\right)$ and their conjugate momenta $\left(\pi_{1}, \pi_{2}\right)$. The integral over $\tau$ runs from 0 to $\beta$ since we are working at finite temperature in the Matsubara imaginary time formalism [16]. The Hamiltonian density for the model is given by

$$
\mathcal{H}=\frac{1}{2}\left(\pi_{i} \pi_{i}+\nabla \phi_{i} \cdot \nabla \phi_{i}\right)+V_{0}+\frac{J_{i}}{\beta \Omega} \phi_{i}
$$

with $\phi^{2} \equiv \phi_{1}^{2}+\phi_{2}^{2}, V_{0}$ is a quadratic potential. This Hamiltonian is invariant under a global $S O(2)$ symmetry which is generated by the integral over all space of the charge density $Q \equiv \phi_{1} \pi_{2}-\phi_{2} \pi_{1}$. The $\pi_{i}$ integrals are easily done and we can rewrite $Z\left[J_{i}\right]$ as follows,

$$
Z\left[J_{i}\right]=N \int \mathcal{D} \phi_{1} \mathcal{D} \phi_{2} \exp \left(-S\left[\phi_{i}, J_{i}\right]\right)
$$

where $N$ is a constant and $S\left[\phi_{i}, J_{i}\right]$ is given by

$$
\begin{aligned}
S\left[\phi_{i}, J_{i}\right] & =\int_{0}^{\beta} d \tau \int d^{3} x\left[\frac{1}{2}\left[\dot{\phi_{1}^{2}}+\dot{\phi}_{2}^{2}+\left(\nabla \phi_{1}\right)^{2}+\left(\nabla \phi_{2}\right)^{2}\right]\right. \\
& \left.+V_{0}(\phi)+\frac{J_{i} \phi_{i}}{\beta \Omega}+i \mu\left(\phi_{2} \dot{\phi_{1}}-\phi_{1} \dot{\phi_{2}}\right)-\frac{\mu^{2} \phi^{2}}{2}\right] .
\end{aligned}
$$

The last two terms of Eq. (19) represent the effects of the conserved charge. We note that $\mu^{2}$ serves as a negative mass squared term, or in other words, we can expect spontaneous symmetry breaking when $\mu$ is greater than the mass of $\phi$. Performing the same calculations that were made in details in sections $I I$ and $I I I$ of Ref. [14], we obtain the one-loop effective potential

$$
V_{\text {eff }}=\frac{\text { const }}{\beta \Omega}+V_{\text {tree }}+V_{\text {thermal }}+V_{\text {zeropoint }},
$$

where

$$
V_{\text {tree }}=V(\phi)-\frac{1}{2} \mu^{2} \phi^{2}+\mu \frac{\bar{Q}}{\Omega}
$$

$$
\begin{aligned}
V_{\text {thermal }} & =\frac{1}{\beta} \int \frac{d^{3} k}{(2 \pi)^{3}} \ln \left\{1-\exp \left[-\beta E_{+}(k)\right]\right\} \\
& \times\left\{1-\exp \left[-\beta E_{-}(k)\right]\right\}
\end{aligned}
$$

and the zero point energy is given by

$$
V_{\text {zeropoint }}=\int \frac{d^{3} k}{(2 \pi)^{3}} \frac{E_{+}(k)+E_{-}(k)}{2},
$$

where the excitation energies are given by

$$
\begin{aligned}
E_{ \pm} & \equiv k^{2}+\mu^{2}+\frac{1}{2}\left(V^{\prime \prime}+\frac{V^{\prime}}{\phi}\right) \\
& \pm \sqrt{2 \mu^{2}\left(2 k^{2}+V^{\prime \prime}+\frac{V^{\prime}}{\phi}\right)+\frac{1}{4}\left(V^{\prime \prime}-\frac{V^{\prime}}{\phi}\right)^{2}}
\end{aligned}
$$

The renormalization can be defined by introduction of counterterms as usual. Below we will quote directly the expression for the renormalized $V_{\text {eff }}$.

The effective potential is one adequate quantity to extract information of the phase structure (in terms of the temperature and chemical potential) and to determine the symmetry changing phase transitions. In the high temperature limit we neglect the contribution $V_{\text {zeropoint }}$, which can be justified as follows. The zero point energy term has no temperature dependence explicitly, but at high $T$ this term will become temperature dependent due to mass renormalization, but this temperature dependence in this limit remains $\lambda$ suppressed relative to the contribution of the $V_{\text {thermal }}$. So we will neglect $V_{\text {zeropoint }}$ in our analysis of the effects of finite charge in the phase structure.

Up to now we have not specified the form of the potential. For simplicity, we will use from now on the $\lambda \phi^{4}$ potential, 


$$
V(\phi)=\frac{1}{2} m^{2} \phi^{2}+\frac{\lambda}{4 !} \phi^{4}
$$

Working with perturbation theory and requiring it to converge, requires that we restrict the the values allowed for the field $\phi$, such that, using one naive criterium, that

$$
\frac{\lambda \phi^{2}}{m^{2}-\mu^{2}} \ll 1 .
$$

The information about the phase structure is in the $\phi$ and $\mu$ dependence of the effective potential. The effective potential is then minimized with respect to the expectation value of the scalar field and the chemical potential. The high temperature effective potential for a $S O(2)$ symmetry at one-loop order is given by

$$
\begin{aligned}
V_{\mathrm{eff}} & =\frac{1}{2}\left(m^{2}-\mu^{2}\right) \phi^{2}+\frac{\lambda}{4 !} \phi^{4}+\mu n-\frac{\pi^{2} T^{2}}{45} \\
& +\frac{T^{2}}{12}\left(m^{2}+\frac{\lambda \phi^{2}}{3}\right)-\frac{\mu^{2} T^{2}}{6}
\end{aligned}
$$

where $n=\frac{\bar{Q}}{\Omega}$ is the charge density.

The phase structure depends on the minima of the effective potential. Minimizing the effective potential with respect the expectation value of the scalar field we obtain

$$
\frac{\partial V_{\mathrm{eff}}}{\partial \phi}=\left(m^{2}-\mu^{2}+\frac{\lambda \phi}{6}+\frac{\lambda T^{2}}{18}\right) \phi .
$$

From Eq. (28), we obtain two minima: from the unbroken symmetry, $\phi=0$, while for the broken symmetry,

$$
\phi^{2}=\left[-m^{2}+\mu^{2}-\frac{\lambda T^{2}}{18}\right] .
$$

From the above equation we obtain the critical temperature $T_{c}$ at which the vacuum expectation value vanishes,

$$
T_{c}^{2}=\frac{18}{\lambda}\left(\mu^{2}-m^{2}\right) .
$$

The relation between $n$ and $\mu$ follows from minimizing the effective potential with respect to $\mu$,

$$
n=\mu \phi^{2}-\frac{\partial V_{\text {thermal }}}{\partial \mu} .
$$

Again, we can neglect the zero point contribution. In the high temperature limit the sum of the two contributions in Eq. (31) becomes

$$
n=\mu \phi^{2}-\frac{\mu T^{2}}{3}
$$

One of our motivations for this work is the application of this formalism in cosmology, as the universe expands at constant entropy. Using the effective potential we can evaluate the entropy density $s=-\frac{\partial V_{\text {eff }}}{\partial T}$. So, as in a expanding universe the charge per comoving volume remains constant, in our analysis we also consider constant volume. Keeping $n / s$ constant, we must require [14]

$$
n=\eta T^{3}
$$

Now we can write $n$ as a function of $\mu, n=f(\mu)$, and then by using Eqs. (29) and (32) we obtain that

$$
n=\frac{6 \mu}{\lambda}\left(\mu^{2}-m^{2}\right)
$$

The analysis of Eq. (34) can be simplified if we work in two limits: at low density $\lambda n \ll m^{3}$ and at high density $\lambda n \gg m^{3}$. The high density limit is particularly more interesting since in this regime $n$ and $\mu$ are large and their effects on the phase structure of the theory is extreme.

From Eq. (34) we get

$$
\mu=\left(\frac{\lambda n}{6}\right)^{1 / 3} \gg m .
$$

Using Eq. (30) we obtain

$$
T_{c}^{2}=3\left(\frac{6 n}{\lambda}\right)^{1 / 3} \gg 18 \frac{\left|m^{2}\right|}{\lambda} .
$$

We see that symmetry breaking occurs much earlier at a much higher temperature, than it would in absence of $n$. Next, we show some numerical results for the high density limit that follows from the analysis of the temperature and density dependent effective potential.

\subsection{Numerical Results}

We here concentrate on the behavior of the $\lambda \phi^{4}$ theory with a charge $n=\eta T^{3}$. In Fig. (1) we see that for a small charge the spontaneously broken symmetry gets restored, as expected, at high temperatures (here we consider $\eta=0.01$ ). But when the charge increases (increasing the value of $\eta$ ) the symmetry seems never to get restored, thus given a symmetry nonrestoration phenomenon analogous as the one seen in the twofield case at finite temperature only, shown in the previous section.

In Fig. (2) we show the behavior of the chemical potential and the expectation value of the scalar field $\phi$ as a function of the temperature as the number density varies, with $n=\eta T^{3}$. We note that this case of unbroken symmetry, we can have a broken phase developing as we increase the density. We can then conclude that a high charge density can induce a high temperature symmetry breaking (or ISB). The symmetry broken solution is not necessarily valid near $T_{c}$ [14], but holds only asymptotically at temperatures when $\mu^{2} \gg m^{2}$. 


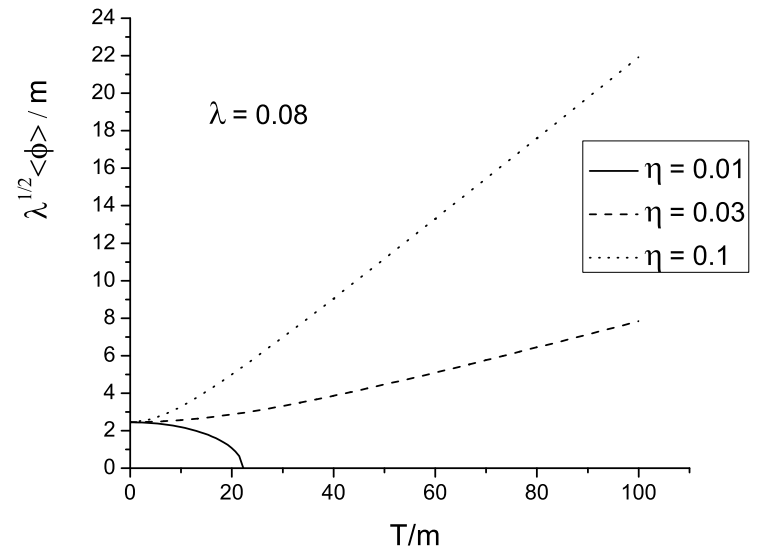

FIG. 1: Behavior of the expectation value of the scalar field $\langle\phi\rangle$ as a function of the temperature $T$ for different values of $\eta$.

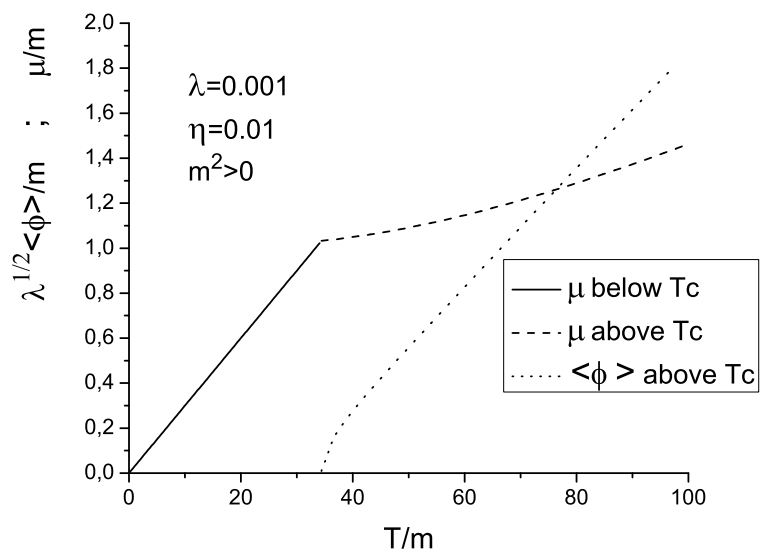

FIG. 2: Behavior for the expectation value of the scalar field $\langle\phi\rangle$ and chemical potential $\mu$ as functions of the temperature $T$, in the unbroken phase.

\section{MULTI-SCALAR FIELD THEORY AT FINITE $T$ AND $\mu$}

By extending our calculations for a coupled two complex scalar fields $\phi$ and $\psi$, we can consider the Lagrangian density,

$$
\begin{aligned}
\mathcal{L}\left(\phi, \phi^{*}, \psi, \psi^{*}\right) & =\left(\partial_{\mu} \phi\right)\left(\partial_{\mu} \phi\right)^{*}-m_{\phi}^{2}\left(\phi \phi^{*}\right)-\frac{\lambda_{\phi}}{3 !}\left(\phi \phi^{*}\right)^{2} \\
& +\left(\partial_{\mu} \psi\right)\left(\partial_{\mu} \psi\right)^{*}-m_{\psi}^{2}\left(\psi \psi^{*}\right) \\
& -\frac{\lambda_{\psi}}{3 !}\left(\psi \psi^{*}\right)^{2}-\lambda\left(\phi \phi^{*}\right)\left(\psi \psi^{*}\right)
\end{aligned}
$$

We can write our expressions in terms of fields $\phi_{i}$ and $\psi_{i}(i=$ 1,2 ) using the transformations

$$
\begin{aligned}
\phi & =\frac{1}{\sqrt{2}}\left(\phi_{1}+i \phi_{2}\right) \\
\psi & =\frac{1}{\sqrt{2}}\left(\psi_{1}+i \psi_{2}\right)
\end{aligned}
$$

In the Grand Canonical formalism, we introduce the chemical potentials $\mu_{\phi}$ and $\mu_{\psi}$ for charge conservation used for each field separately. The resulting action becomes

$$
\begin{aligned}
S & =\int_{0}^{\beta} d \tau \int d^{3} x\left[\frac{1}{2}\left[\dot{\phi}_{1}^{2}+\dot{\phi}_{2}^{2}+\left(\nabla \phi_{1}\right)^{2}+\left(\nabla \phi_{2}\right)^{2}\right]\right. \\
& +\frac{\lambda}{4 !}\left(\phi_{1}^{2}+\phi_{2}^{2}\right)^{2}+\frac{J_{i} \phi_{i}}{\beta \Omega}+i \mu_{\phi}\left(\phi_{2} \dot{\phi}_{1}-\phi_{1} \dot{\phi}_{2}\right) \\
& -\frac{\mu_{\phi}^{2}}{2}\left(\phi_{1}^{2}+\phi_{2}^{2}\right)+\frac{1}{2}\left[\dot{\psi}_{1}^{2}+\dot{\psi}_{2}^{2}+\left(\nabla \psi_{1}\right)^{2}+\left(\nabla \psi_{2}\right)^{2}\right] \\
& +\frac{\lambda}{4 !}\left(\psi_{1}^{2}+\psi_{2}^{2}\right)^{2}+\frac{J_{i} \psi_{i}}{\beta \Omega}+i \mu_{\psi}\left(\psi_{2} \dot{\psi}_{1}-\psi_{1} \dot{\psi}_{2}\right) \\
& \left.-\frac{\mu_{\psi}^{2}}{2}\left(\psi_{1}^{2}+\psi_{2}^{2}\right)+\frac{\lambda}{4}\left(\phi_{1}^{2}+\phi_{2}^{2}\right)\left(\psi_{1}^{2}+\psi_{2}^{2}\right)\right] .
\end{aligned}
$$

The next step consist in expanding the action to determine a expression for the effective potential. From Eq. (39) and following similar computation of the effective potential as for the one-field case, the one-loop effective potential is determined by the functional partition function to one-loop order and given by

$$
\begin{aligned}
V_{\mathrm{eff}}(\phi, \psi) & =\frac{m_{\phi}^{2}}{2}\left(\phi_{1}^{2}+\phi_{2}^{2}\right)+\frac{\lambda_{\phi}}{4 !}\left(\phi_{1}^{2}+\phi_{2}^{2}\right)^{2} \\
& +\frac{m_{\psi}^{2}}{2}\left(\psi_{1}^{2}+\psi_{2}^{2}\right)+\frac{\lambda_{\psi}}{4 !}\left(\psi_{1}^{2}+\psi_{2}^{2}\right)^{2} \\
& +\frac{\lambda}{4}\left(\phi_{1}^{2}+\phi_{1}^{2}\right)\left(\psi_{1}^{2}+\psi_{2}^{2}\right)+\frac{1}{2} \operatorname{Tr} \ln \hat{M}
\end{aligned}
$$

where $\hat{M}$ is the matrix operator for the quadratic terms in the fluctuations, 


$$
\hat{M}=\left(\begin{array}{cccc}
-\partial_{\mu} \partial_{\mu}-\mu^{2}+U_{11}^{\phi} & 2 i \mu \frac{\partial}{\partial \tau}+\frac{\lambda_{\phi}}{3} \phi_{1} \phi_{2} & \lambda \phi_{1} \psi_{1} & \lambda \phi_{1} \psi_{2} \\
-2 i \mu \frac{\partial}{\partial \tau}+\frac{\lambda_{\phi}}{3} \phi_{1} \phi_{2} & -\partial_{\mu} \partial_{\mu}-\mu^{2}+U_{22}^{\phi} & \lambda \phi_{2} \psi_{1} & \lambda \phi_{2} \psi_{2} \\
\lambda \psi_{1} \phi_{1} & \lambda \psi_{1} \phi_{2} & -\partial_{\mu} \partial_{\mu}-\mu^{2}+U_{33}^{\psi} & 2 i \mu \frac{\partial}{\partial \tau}+\frac{\lambda \psi}{3} \psi_{1} \psi_{2} \\
\lambda \psi_{1} \phi_{2} & \lambda \psi_{2} \phi_{2} & -2 i \mu \frac{\partial}{\partial \tau}+\frac{\lambda_{\psi}}{3} \psi_{1} \psi_{2} & -\partial_{\mu} \partial_{\mu}-\mu^{2} U_{44}^{\psi}
\end{array}\right),
$$

where

$$
\begin{aligned}
U_{11}^{\phi} & =m_{\phi}^{2}+\frac{\lambda_{\phi}}{2} \phi_{1}^{2}+\frac{\lambda_{\phi}}{6} \phi_{2}^{2}+\frac{\lambda}{2} \psi^{2}, \\
U_{22}^{\phi} & =m_{\phi}^{2}+\frac{\lambda_{\phi}}{2} \phi_{2}^{2}+\frac{\lambda_{\phi}}{6} \phi_{1}^{2}+\frac{\lambda}{2} \psi^{2}, \\
U_{33}^{\psi} & =m_{\psi}^{2}+\frac{\lambda_{\psi}}{2} \psi_{1}^{2}+\frac{\lambda_{\psi}}{6} \psi_{2}^{2}+\frac{\lambda}{2} \phi^{2}, \\
U_{44}^{\psi} & =m_{\psi}^{2}+\frac{\lambda_{\psi}}{2} \psi_{2}^{2}+\frac{\lambda_{\psi}}{6} \psi_{1}^{2}+\frac{\lambda}{2} \phi^{2} .
\end{aligned}
$$

Minimizing the effective potential in relation to $\phi$ and $\psi$, the phase structure for this theory can be determined. According to the results of Sec. II.B, the two-field case, for a convenient choice of couplings, can result naturally in the phenomena of ISB/SNR. From the discussion and analysis of the effects of a finite charge (density) these symmetry change effects can appear even in the one-field case. Thus, we expect that these density effects included in Eq. (40) will strength the emergence of such phenomena in the two-field, or multi-field models. An extensive analysis of the resulting phase diagram obtained from Eq. (40) will be shown elsewhere [17].

\section{CONCLUSIONS}

In this work we have shown that a high charge density can induce strong changes in the phase structure of the theory. Be- sides high temperature symmetry restoration it can also exhibit symmetry nonrestoration and inverse symmetry breaking. We can look for this phenomena in different branches of physics, like condensed matter, cosmology and even in applications motivated by the up to date heavy-ion collision experiments. Since phenomena like ISB/SNR can appear in a theory with one self-interacting complex scalar field at finite density, it is an interesting matter to explore the same phase structure behavior in models with higher field content. We are currently looking for this kind of phenomena in a multi-scalar field theory at finite temperatures and densities [17].

As a future application we intend to perform the dynamics of the multi-scalar field model in order to probe, dynamically, the emergence of such interesting phase behaviors like ISB/SNR. One step toward this comparison was done in the classical level for the self-interacting scalar field theory in Ref. [18].

\section{Acknowledgments}

The authors would like to thank FAPERJ, CNPq and CAPES for the financial support.
[1] C. W. Bernard, Phys. Rev. D. 9, 3312 (1974).

[2] L. Dolan and R. Jackiw, Phys. Rev. D 9, 3320 (1974).

[3] J. Bernstein and S. Dodelson, Phys. Rev. Lett. 66, 683 (1991).

[4] R. L. S. Farias, G. Krein, and R. O. Ramos, arXiv:0809.1449 (in press Phys. Rev. D).

[5] A. Linde, Rep. Prog. Phys. 42, 389 (1979).

[6] S. Weinberg, Phys. Rev. D 9, 3357 (1974).

[7] R. O. Ramos and M. B. Pinto, Phys. Rev. D 61, 125016 (2000).

[8] M. B. Pinto, R. O. Ramos, and J. E. Parreira Phys. Rev. D 71, 123519 (2005).

[9] R. N. Mohapatra and G. Senjanovic Phys. Rev. Lett. 42, 1651 (1979); S. Dodelson and L. M. Widrow, Phys. Rev. Lett. 64, 340 (1990); B. Bajc and G. Senjanovic, Nucl. Phys. Proc. Suppl. A 52, 246 (1997).

[10] N. Schupper and N. M. Shnerb, Phys. Rev. E 72, 046107 (2005).
[11] R. O. Ramos and M. B. Pinto, J. Phys. A 39, 6687 (2006).

[12] M. B. Pinto, R. O. Ramos, and F. F. de Souza Cruz, Phys. Rev. A 74, 033618 (2006).

[13] R. O. Ramos and M. B. Pinto, J. Phys. A 39, 6649 (2006).

[14] K. M. Benson, J. Bernstein, and S. Dodelson, Phys. Rev. D 44, 2480 (1991).

[15] M. B. Pinto and R. O. Ramos, Phys. Rev. D 61, 125016 (2000).

[16] J. I. Kapusta, Phys. Rev. D 24, 426 (1981); H. E. Haber and H. Weldon, Phys. Rev. Lett. 46, 1497 (1981); Phys. Rev. D 25, 502 (1982); J. I. Kapusta, Finite Temperature Field Theory (Cambridge University Press, Cambridge, England, 1989).

[17] R. L. S. Farias, R. O. Ramos, and R. Vartuli, in preparation.

[18] R. L. S. Farias, R. O. Ramos, and L. A. da Silva, Langevin Simulations with Colored Noise and Non-Markovian Dissipation, in press Braz. J. Phys., 38, 3B, (2008). 